\title{
Diagnosis and Reporting of Follicular-Patterned Thyroid Lesions by Fine Needle Aspiration
}

\author{
William C. Faquin
}

Received: 27 December 2008/ Accepted: 21 January 2009/Published online: 22 February 2009

(c) Humana 2009

\begin{abstract}
Over the past 3 decades, fine needle aspiration (FNA) has developed as the most accurate and costeffective initial method for guiding the clinical management of patients with thyroid nodules. Thyroid FNA specimens containing follicular-patterned lesions are the most commonly encountered and include various forms of benign thyroid nodules, follicular carcinomas, and the follicular variant of papillary thyroid carcinoma. Based primarily upon the cytoarchitectural pattern, FNA is used as a screening test for follicular-patterned lesions to identify the majority of patients with benign nodules who can be managed without surgical intervention. The terminology and reporting of thyroid FNA results have been problematic due to significant variation between laboratories, but the recent multidisciplinary NCI Thyroid FNA State of the Science Conference has provided a seven-tiered diagnostic solution. A key element of this approach is the category "atypical cells of undetermined significance" (ACUS) which is used for those aspirates which cannot be easily classified as benign, suspicious, or malignant. Lesions in this category represent approximately $3-6 \%$ of thyroid FNAs and have a risk of malignancy intermediate between the "benign" category and the "suspicious for a follicular neoplasm" category. The recommended follow-up for an ACUS diagnosis is clinical correlation and in most cases, repeat FNA sampling.
\end{abstract}

Keywords Thyroid · FNA · Follicular · Bethesda

W. C. Faquin $(\square)$

Department of Pathology, WRN 219, Massachusetts General

Hospital and Harvard Medical School, 55 Fruit Street,

Boston 02114, MA, USA

e-mail: WFaquin@Partners.org

\section{Introduction}

In many pathology practices, thyroid FNAs comprise a significant proportion of non-gynecologic cytology specimens reflecting the fact that nodules of the thyroid gland are very common. It is estimated that as many as $4-7 \%$ of the adult population has a palpable thyroid enlargement, and up to 10 times this number of individuals have subclinical nodules [1-4]. The majority of these thyroid nodules are benign with only a fraction representing malignant disease which accounts for approximately $1.1 \%$ of all cancers annually [5]. The large number of benign thyroid nodules relative to the small number of malignant ones creates a clinical dilemma-how to manage patients with a detectable thyroid enlargement that statistically is more likely to be benign? Over the past three decades, FNA has developed as the most accurate and cost-effective initial method for evaluating thyroid nodules [6]. In experienced hands, the diagnostic accuracy of thyroid FNA for technically satisfactory specimens ranges from 80 to $>95 \%$ [7].

FNA can be used as either a screening test for follicular carcinoma, or as a diagnostic test for other thyroid carcinomas including papillary carcinoma, medullary carcinoma, undifferentiated carcinoma, and lymphoma. The follicular-patterned lesion is the most commonly encountered type of thyroid FNA specimen, and it will be the focus of this discussion. Follicular-patterned lesions include benign thyroid nodules (adenomatous nodules, hyperplastic nodules, follicular adenomas), follicular carcinomas, and the follicular variant of papillary thyroid carcinoma. The application of thyroid FNA in the evaluation of the various follicular-patterned lesions, including key diagnostic pitfalls, and recent advances by the NCI Thyroid FNA State of the Science Conference in the reporting of these lesions will be reviewed. 


\section{Discussion}

FNA as a Screening Test for Follicular Carcinoma

The application of FNA to distinguish benign follicular nodules from follicular carcinomas is problematic because the criteria for distinguishing between them are based upon histologic evidence of transcapsular or vascular invasion. Since invasion cannot be assessed by FNA, the question becomes "how can FNA be used to evaluate follicular neoplasia." The answer is that FNA is used as a screening test for follicular carcinoma. The cytologic criteria used to distinguish benign from suspicious thyroid lesions includes the follicular group architecture, smear cellularity, amount of colloid, and cytologic atypia [8]. By far, the most important of these criteria is cytoarchitecture $[9,10]$. Thus, while a specific diagnosis may not be given, it is possible to subcategorize these lesions into two groups: those which are almost certainly benign, and those which are suspicious for a follicular neoplasm and possibly malignant. This subcategorization identifies a majority of patients with benign lesions for whom surgical intervention can usually be avoided.

Benign thyroid nodules are characterized microscopically by a macrofollicular pattern which features large flat sheets of follicular cells with small dark round nuclei in a honeycomb arrangement, usually in a background of moderate to abundant colloid (Fig. 1). The flat sheets of cells result from fragmentation of macrofollicles with extrusion of colloid during the smear preparation. In contrast, thyroid aspirates composed of microfollicles (small follicular groups of 6-12 follicular cells in a ring with or without a small amount of central colloid), crowded trabeculae, or 3-D groups of overlapping follicular cells are a

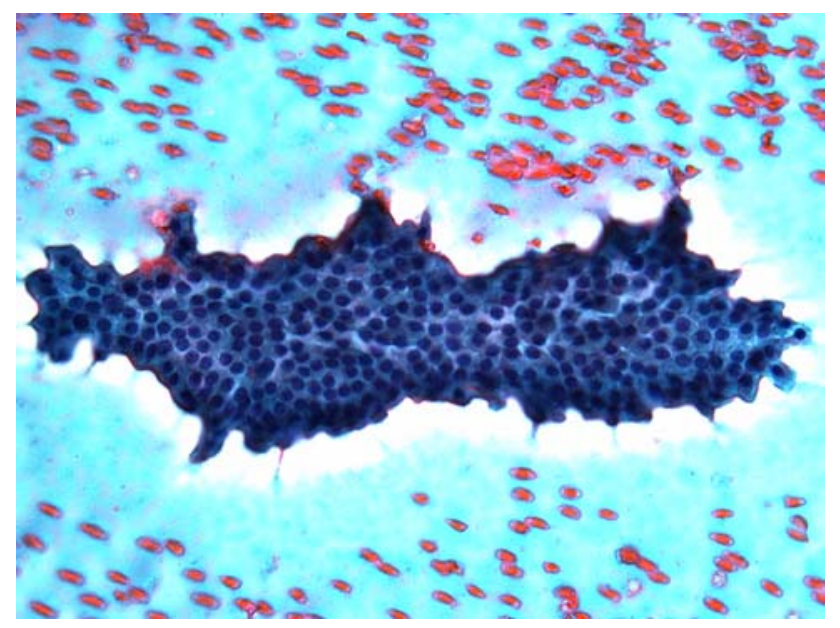

Fig. 1 Thyroid FNA demonstrating a macrofollicular group of cells in a background of abundant watery colloid. These features are typical of a benign thyroid nodule. (Papanicolaou stain) feature of follicular carcinomas as well as some adenomas (Fig. 2). These aspirates are diagnosed as "suspicious for a follicular neoplasm," and it is this group of patients for whom thyroid lobectomy is generally considered warranted.

By sorting follicular lesions into two groups based primarily upon cytoarchitecture, FNA can identify a large subset of nodules that are benign, and that do not require surgery. This approach works because follicular carcinomas are rarely composed of predominantly normal-sized or macrofollicles $[8,10]$. The key to using this approach is to focus upon the predominant cytoarchitectural pattern. A common pitfall is to identify a minor population of microfollicles and ignore the overall pattern. In addition, it is important to also evaluate the nuclear features of the cells to avoid the pitfall of the follicular variant of papillary thyroid carcinoma.

Unfortunately, while FNA as a screening test is highly sensitive, it lacks specificity. Approximately $15-30 \%$ of aspirates diagnosed as "suspicious for a follicular neoplasm" are actually carcinomas while the remaining 70 $85 \%$ of nodules are benign. [11-13] A wide variety of immunohistochemical and molecular markers have been studied in an attempt to distinguish between benign thyroid nodules and follicular carcinomas in FNA samples; however, to date, none has been identified with both high sensitivity and specificity. Until such marker is available, FNA will remain a screening test rather than a diagnostic test for follicular carcinomas.

\section{The Follicular Variant of Papillary Thyroid Carcinoma}

The follicular variant of papillary thyroid carcinoma is the most common subtype of papillary thyroid carcinomas [5].

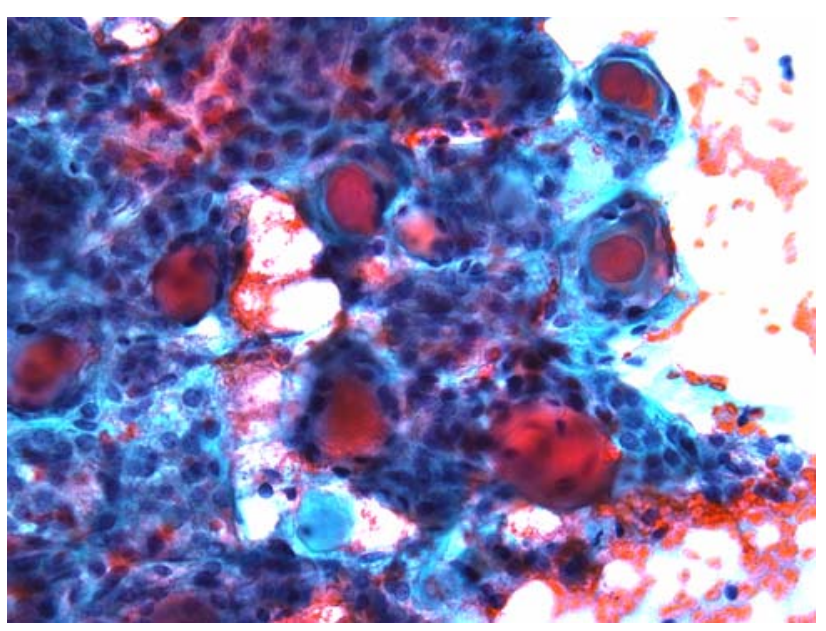

Fig. 2 Thyroid FNA showing follicular cells in a predominantly microfollicular arrangement. This cytoarchitectural pattern is characteristic of a follicular neoplasm. (Papanicolaou stain) 
In FNA specimens, it can pose a diagnostic challenge due to the abundance of microfollicles or monolayer tissue fragments mimicking a follicular neoplasm. In fact, among those FNAs diagnosed as "suspicious for a follicular neoplasm" that are malignant in histologic follow-up, over $30 \%$ are identified as the follicular variant of papillary thyroid carcinoma $[13,14]$. In contrast to the hyperchromatic chromatin seen in follicular neoplasms, the follicular variant of papillary thyroid carcinoma exhibits pale, powdery chromatin along with nuclear grooves and occasional intranuclear pseudoinclusions (Fig. 3). The cells tend to be round to oval and less pleomorphic than the conventional type of papillary carcinoma. Variable amounts of dense colloid are frequently seen in this variant, and multinucleated giant cells may also be identified. Usually the cytologic diagnosis of the follicular variant of papillary carcinoma is not difficult, but in a subset of cases, the nuclear features can be quite subtle resulting in misclassification of the lesion. Development of methods to detect RET/PTC rearrangements or point mutations of the BRAF gene in routine thyroid FNA samples may prove useful for solving this problem [15].

\section{Reporting of Thyroid FNAs}

A major challenge in the application of FNA to the diagnosis of thyroid lesions has been the inconsistent use of terminology for reporting results of thyroid FNAs both within laboratories and between different institutions. Not only has this hindered the sharing of information between different institutions, but it has created difficulties for clinicians managing patients with thyroid disease. A major advance on this front was made by the NCI Thyroid FNA State of the Science Conference which convened on October 22-23,

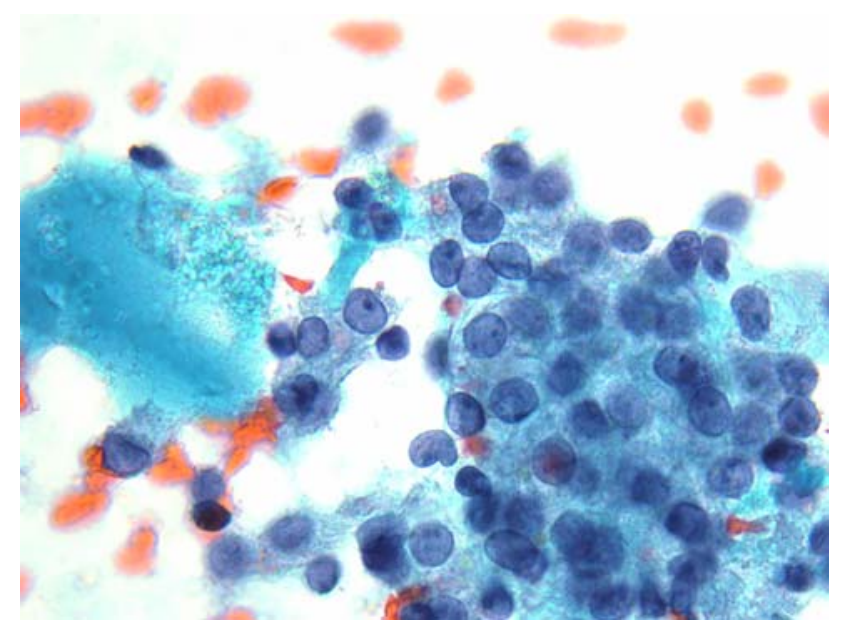

Fig. 3 The follicular variant of papillary thyroid carcinoma is characterized by cells with pale chromatin, somewhat enlarged oval nuclei, and occasional longitudinal nuclear grooves in a background of variable amounts of colloid. (Papanicoloau stain)
2007 in Bethesda, Maryland. It addressed issues including indications for thyroid FNA, training, techniques, reporting terminology, ancillary testing, and management guidelines $[16,17]$. Based upon the findings, a Bethesda System-type diagnostic framework for reporting thyroid FNA results was proposed that consists of seven general diagnostic categories (Table 1). Importantly, the terms used for reporting results are associated with a risk of malignancy which will impact recommendations for patient management [16].

\section{Atypical Cells of Undetermined Significance (ACUS)}

While most follicular-patterned lesions can be accurately assessed using thyroid FNA, a small subset of lesions exhibit cytologic features that are intermediate between benign and neoplastic. This so-called "indeterminate" category has been used in various ways by different laboratories. Some have used this category for any aspirate with atypia, for others the category has been used to mean "indeterminate for malignancy" or "indeterminate for neoplasia." In the proposed Bethesda System for thyroid cytology, the ACUS or indeterminate category is specifically designated for use when the 'cytologic and/or architectural atypia encountered is of uncertain significance: it is of an insufficient degree to qualify for any of the suspicious or malignant categories' [18]. At the same time, the cytologic or architectural atypia is more than would be acceptable within the "benign" category. There are many different situations in which an ACUS diagnosis would be used, but some of the most common include the following: (1) a sparsely cellular aspirate with a predominance of microfollicles, (2) cytologic atypia in the setting of preparation artifact, (3) a mixed cytoarchitectural pattern that includes nearly equal proportions of macrofollicles and microfollicles (Fig. 4), (4) focal atypia suggestive of papillary carcinoma in an otherwise predominantly benign-appearing sample (Fig. 5). Because of the potential to overuse the ACUS category, it should be less than $7 \%$ of all thyroid FNA interpretations [18]. For thyroid aspirates diagnosed as ACUS, the recommended management is clinicoradiologic correlation and in most cases, a repeat

Table 1 Proposed 7-tiered Bethesda system for reporting of thyroid cytology $^{\mathrm{a}}$

\begin{tabular}{ll}
\hline Diagnostic category & Risk of malignancy $(\%)$ \\
\hline Insufficient for diagnosis & $1-4$ \\
Benign & $<1$ \\
Atypical cells of undetermined significance & $5-10$ \\
Suspicious for a follicular neoplasm & $15-30$ \\
Suspicious for a Hurthle cell neoplasm & $15-45$ \\
Suspicious for malignancy & $60-75$ \\
Malignant & $97-99$
\end{tabular}

${ }^{a}$ Modified from Cibas and Ali 2009 


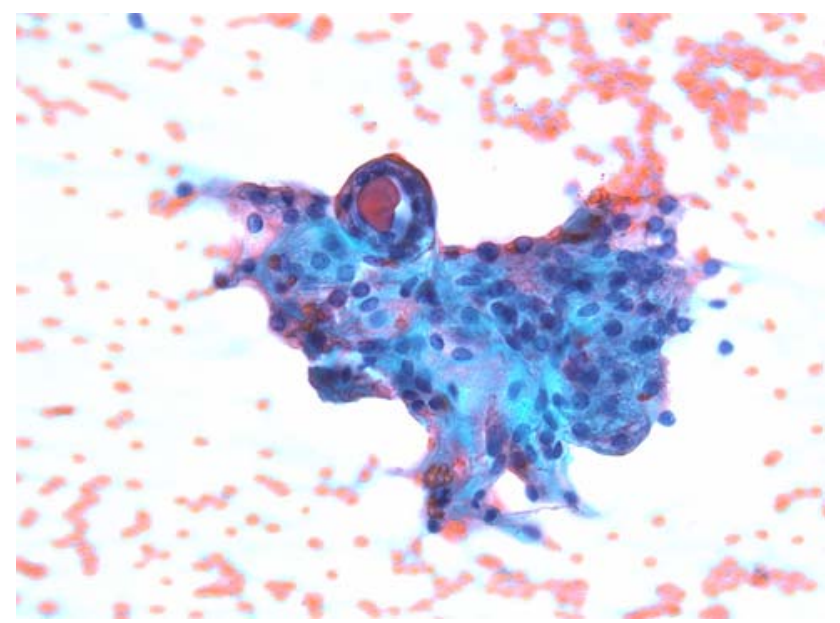

Fig. 4 This thyroid FNA demonstrates an indeterminate architectural pattern and is placed into the category "Atypical cells of undetermined significance (ACUS)." (Papanicolaou stain)

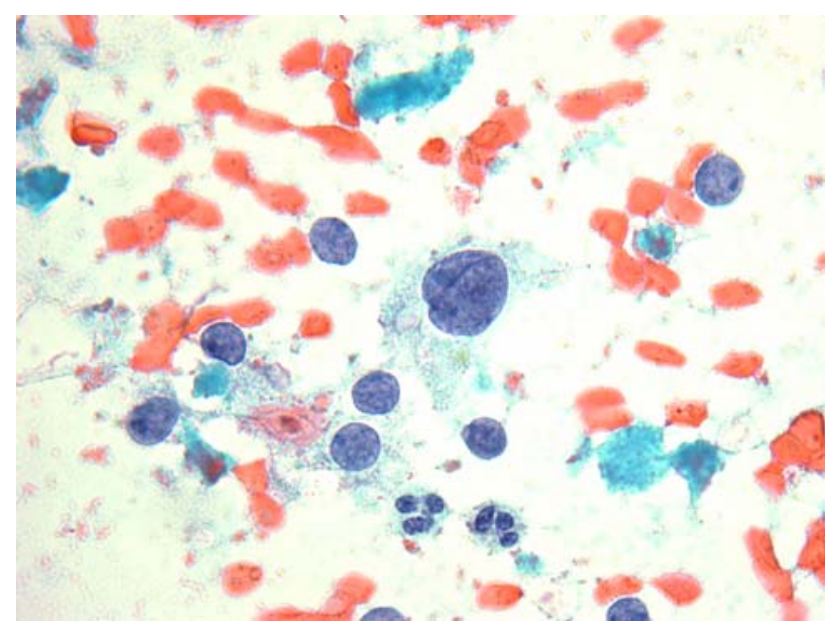

Fig. 5 This thyroid FNA contains occasional follicular cells with enlarged, pale, grooved nuclei in an otherwise benign aspirate, and is therefore diagnosed as "Atypical cells of undetermined significance (ACUS)." (Papanicoloau stain)

FNA. The latter will often result in a more definitive "benign," "suspicious," or "malignant" designation; however, 20-25\% of cases will result in a second ACUS diagnosis $[13,18]$. The risk of malignancy for an ACUS diagnosis is difficult to assess because only a subset of ACUS nodules will be surgically excised. However, it is estimated that the overall risk of malignancy will be approximately $5-10 \%$ which is intermediate between the "benign" and "suspicious for a follicular neoplasm" categories [18].

\section{Conclusions}

Follicular-patterned thyroid lesions are a common and sometimes challenging area of cytopathology. FNA has developed as an effective means for evaluating these lesions particularly when combined with the recently proposed Bethesda System for reporting of thyroid FNAs. An important advance in this regard is the use of diagnostic categories with an associated risk of malignancy upon which patient management decisions can be based.

\section{References}

1. Stoffer RP, Welch JW, Hellwig CA, Chesky VE, McCusker EN. Nodular goiter: incidence, morphology before and after iodine prophylaxis, and clinical diagnosis. Arch Intern Med. 1960;106: $10-4$.

2. Klonoff DC, Greenspan FS. The thyroid nodule. Adv Intern Med. 1982;27:101-26.

3. Vander JB, Gaston EA, Dawber TR. The significance of nontoxic thyroid nodules. Final report of a 15-year study of the incidence of thyroid malignancy. Ann Intern Med. 1968;69:537-40.

4. Rojeski MT, Gharib H. Nodular thyroid disease. Evaluation and management. N Engl J Med. 1985;313:428-36.

5. DeLellis RA, Lloyd RV, Heitz PU, Eng C. World health organization classification of tumors: tumours of endocrine organs. Lyon: IARC Press; 2004.

6. The American Thyroid Association Guidelines Taskforce. Management guidelines for patients with thyroid nodules and differentiated thyroid cancer. Thyroid. 2006;16:1-33.

7. Yang J, Schnadig V, Logrono R, Wasserman PG. Fine-needle aspiration of thyroid nodules: a study of 4703 patients with histologic and clinical correlations. Cancer Cytopathol. 2007;111: 306-15.

8. Clark DP, Faquin WC. Thyroid cytopathology. New York: Springer; 2005.

9. Gardner HA, Ducatman BS, Wang HH. Predictive value of fine needle aspiration of the thyroid in the classification of follicular lesions. Cancer. 1993;71:2598-603.

10. Cibas ES, Ducatman BS. Cytology: diagnostic principles and clinical correlates. New York: Saunders; 2009.

11. Gharib H, Goellner JR. Fine-needle aspiration biopsy of the thyroid: an appraisal [see comments]. Ann Intern Med. 1993;118: 282-9.

12. Baloch ZW, Fleisher S, et al. Diagnosis of "follicular neoplasm": a gray zone in thyroid fine-needle aspiration cytology. Diagn Cytopathol. 2002;26:41-4.

13. Yassa L, Cibas ES, Benson CB, et al. Long-term assessment of multidisciplinary approach to thyroid nodule diagnostic evaluation. Cancer Cytopathol. 2007;111:508-16.

14. Deveci MS, Deveci G, LiVolsi VA, Baloch ZW. Fine-needle aspiration of follicular lesions of the thyroid. Diagnosis and follow-up. Cytojournal. 2006;3:1-9.

15. Nikiforov YE. Thyroid carcinoma: molecular pathways and therapeutic targets. Mod Pathol. 2008;21:S37-43.

16. Cibas ES, Ali SZ. The Bethesda system for reporting thyroid cytopathology. New York: Springer; 2009.

17. Baloch ZW, LiVolsi VA. Diagnostic terminology and morphologic criteria for cytologic diagnosis of thyroid lesions: a synopsis of the national cancer institute thyroid fine-needle aspiration state of the science conference. Diagn Cytopathol. 2008;36:425-37.

18. Krane JF, Nayar R, Renshaw AA. Atypical cells of undetermined significance. In: Ali SZ, Cibas ES, editors. The Bethesda system for reporting thyroid cytopathology. New York: Springer; 2009. 